\title{
Débat avec le public
}

\section{(2) OpenEdition \\ 12 Journals}

Édition électronique

URL : http://journals.openedition.org/communicationorganisation/1985

DOI : 10.4000/communicationorganisation. 1985

ISSN : $1775-3546$

Éditeur

Presses universitaires de Bordeaux

\section{Édition imprimée}

Date de publication : 1 novembre 1997

ISSN : 1168-5549

\section{Référence électronique}

"Débat avec le public », Communication et organisation [En ligne], 12 | 1997, mis en ligne le 26 mars

2012, consulté le 20 avril 2019. URL : http://journals.openedition.org/

communicationorganisation/1985; DOI : 10.4000/communicationorganisation.1985

Ce document a été généré automatiquement le 20 avril 2019.

(C) Presses universitaires de Bordeaux 


\section{Débat avec le public}

1 Wolfgag Snettekorn : Je partirai d'un constat de Ferdinand de Saussure qui dit : «c'est le point de vue qui crée l'objet ». Une question qui me tracasse et sur laquelle j'ai travaillé est «qu'est-ce qui crée le point de vue?». Votre intervention pourrait aller dans cette direction mais il y a un facteur qui me semble important et que vous n'avez pas mentionné, un facteur purement matériel et médiatique. Par exemple quand on observe le développement de l'anthropologie, de l'ethnologie et de la démographie, on peut constater que nombre de questions méthodologiques et de conceptions de l'objet proviennent de l'utilisation des médias. Par exemple, les travaux de Goffman et autres méthodologues ont posé les questions de méthodologie à partir de l'utilisation du magnétophone. Toutes les questions de participation se sont posées au moment où il y avait un élément qui intervenait : le micro, la caméra vidéo, l'appareil photo...

2 Pensez-vous que ce soit vraiment un facteur intervenant? J'ajoute, sur le sujet de notre colloque, «induction » : sans électricité il n'y aurait pas un sens de l'induction qui aurait pu être mentionné ici.

3 D'autre part, dans quelle mesure croyez-vous que dans la transparence intervienne une culture qui porte les empreintes religieuses, c'est-à-dire qui porte les empreintes du protestantisme -qui renvoie les sujets à eux-mêmes, à se regarder, à se concevoir transparents vis-à-vis d'un dieu - les empreintes du catholicisme -qui conçoit le moi par une conception de l'église - et les empreintes de la conception juive qui passe par la psychanalyse?

4 Lors d'un colloque à Londres en 1979, auquel participait Goffman, le grand débat était de voir les inductions que ces médiations, la caméra, le magnétophone, la photographie, injectaient dans le processus de recherche. Pour éviter de répondre à cette question, je suis parti de l'idée qu'il s'agissait d'observations à l'œil nu. J'ai opposé cette observation médiate à une observation immédiate, en face à face. Il est vrai que j'ai toujours été formé à ne rien utiliser, ou s'il s'agit d'utiliser l'appareil photo ou l'enregistreur c'est à visage découvert. Mais ici la question est de savoir jusqu'où peut-on se permettre de négocier cette présence ? En tout cas, il va de soi qu'on ne cache pas le matériel (mais cela n'est pas un problème de négociation mais d'éthique), et donc à partir du moment où on décide de montrer l'appareil, on décide de le prendre. Certaines écoles vont dire que l'interlocuteur s'habitue à sa présence et qu'il ne faut pas mystifier les machines. Personnellement, je 
suis de l'autre tendance, qui consiste à penser que plus on est entouré d'objets et plus on se réfugie derrière eux pour se désengager. Donc, ce n'est pas tant le problème du rapport à l'autre, ni le problème du rapport de l'autre à ces machines que j'évoque ici, mais mon propre rapport à ces machines. Il me semble que l'observation est beaucoup plus participante lorsque l'on est seul face à l'autre, éventuellement avec un stylo ou un crayon, et encore, je ne préfère pas. Mais j'insiste alors sur l'écriture «juste-après «. Ma médiation se situe peut-être là. J'ai l'impression aussi que les appareils nous rajoutent du travail. Il faut tout décoder après. C'est un peu comme si vous plongiez dans l'observation, ou même dans la participation, avec toutes vos cassettes qui défilent.

Donc ma réponse est celle-ci, et c'est celle que je donne à mes étudiants : « vous y allez tout nu, je ne veux pas d'appareil».

6 Pour répondre à votre deuxième question, je dirais que le rapport de la judéité à la psychanalyse me rappelle le livre de Coudy Hile qui historiquement essaie de montrer cela : il montre que l'émergence de la psychanalyse et le travail personnel de Freud, le travail de pénétration de la bourgeoisie viennoise permet de lire la psychanalyse comme une autobiographie. J'ai moi-même utilisé cette interprétation pour faire la trajectoire de Goffman. Cette thèse est irritante pour la communauté juive notamment celle des EtatsUnis. Je me rappelle une conversation avec Coudy Hile où il me disait qu'à la sortie de son livre, ce fut le black-out total de la part des médias américains. J'ai l'impression qu'il faut effectivement faire très attention à délimiter des communautés dites catholiques, dites protestantes, dites juives, en ce sens qu'à l'intérieur de chacune il y a des tendances très différentes.

7 Mais il n'empêche, qu'en tant qu'hypothèse générale de travail, elle vaut la peine d'être construite. Elle n'est certainement pas une hypothèse à l'emporte-pièce que l'on peut balayer d'un revers de la main.

8 Pierre Delcambre : Dans le travail présenté sur la participation, il est question du mode de présence à l'autre et du mode de présence à soi. Si un rapport est à faire avec la question de l'induction, on pourrait vraisemblablement imaginer que l'induction pose aussi la question du rapport à la présence du sens. Dans les différents textes qui ont été proposés, certains mettent en jeu l'induction par rapport à un trop-plein de sens, d'autres en revanche sont «pas assez de sens", ou à du «non-sens tout de suite» de l'infracommunication, pour reprendre une des formules. Ce qui m'a intéressé c'est que, dans le parcours cité, notamment le touriste et son double, le mode de participation renvoie au rôle de l'observateur, je me souviens que dans "Anthropologie de la communication ", ce n'est pas seulement sur Goffman que l'on s'appuie mais aussi sur Hymes.

$9 \quad$ Et ce qui est montré dans la reprise du modèle speaking de Hymnes est que, pour les participants, pour un quelconque membre d'une communauté, il se peut qu'il ne se passe rien. Il y a des questions autour de ce phénomène. Mais j'ai envie de travailler sur l'induction : les textes que l'on a lu parlent beaucoup d'ellipse, d'éllipse de sens et aussi de réserve (on ne comprend pas tout de suite). Dès lors que l'on est dans son rôle, donc que l'observateur participant est à sa propre place, qu'en est-il des absences de sens, de ces « trop-plein de sens » que l'on tâche de mettre en réserve ? En fait, comment se situe-t-on par rapport à ce que certains appellent « l'induction discible »?

10 On ne maitrise pas la notion d'induction telle que les chercheurs du Grec/o depuis quelques années l'ont construite. Il est vrai que je n'ai pas voulu faire d'allusion à Hymnes 
qui réduit quelque peu le spectre sémantique de la notion de communication, spectre extrêmement large si l'on suit Bateson. Et en cela Hymnes n'a peut-être pas tort. Il permet de souligner le fait qu'il peut et qu'il doit y avoir des blancs, pour qu'ensuite il puisse y avoir des pleins. Cela peut paraitre idiot de le dire tel quel, mais c'est pourtant de cela qu'il s'agit. En fait, j'ai comme l'impression que l'observateur participant qui se mue en écrivain, ou en écrivant, va essayer de restructurer ces «trop-plein « et ces «troppeu », de les rééquilibrer par l'écriture. Il me semble qu'il y aurait un travail à réaliser qui consisterait à voir ce que font de leurs notes les chercheurs qui sont sur le terrain. $\mathrm{H}$ me semble que par moment ils essaient de vider certaines poches de "trop-plein » et qu'à d'autres moments il y a du «trop-peu » qu'ils ouvrent au maximum. Il y a des moments qui restent tout-à-fait blancs et qu'ils rempliront ensuite d'un commentaire. Donc il y aurait le moyen d'utiliser le modèle de Hymnes pour refaire une exploitation de l'observateur participant tenu, en écrivant, à ses variations de sens, à ses ellipses, travailler sur les points d'interrogation d'un journal de chercheur. Les points d'interrogation correspondraient aux vides, les points d'exclamation seraient les «tropplein «; on ne s'en sortirait plus.

11 C'est d'ailleurs ce que j'essaie de travailler avec de jeunes apprentis anthropologues de la communication à Liège, en leur disant de ne pas céder à la panique et donc, d'une part, de ne pas se dire qu'il y en a trop, essayer d'y aller plus calmement, essayer de faire des petits dessins, de reprendre les postures corporelles, d'appréhender, de maîtriser les «trop-plein ». Et par ailleurs s'ils sentent que rien ne se passe, essayer au contraire de s'engouffrer là-dedans, en posant les questions alimentées par la théorie.

Donc, je crois qu'il y a du «trop-plein » et du «trop-vide», et qu'il y a en même temps une tentative de rééquilibre progressif, parce que le journal s'écrit, se réécrit et se stratifié.

13 Michèle Gabay : Je souhaiterais revenir sur les supports d'observation. Je rebondis sur la question que vous avez posée, relative à l'utilisation du caméscope comme moyen d'observation. J'ai travaillé sur la formation à l'observation en utilisant le magnétoscope dans la situation que vous dépeigniez tout-à-l'heure (celle de la personne qui achète un tapis et qui se laisse induire dans son comportement). Imaginons que l'on ait un caméscope qui aurait filmé. Vous auriez peut-être pu écrire votre article à la lueur du film, et à ce moment il y aurait eu deux phénomènes qui auraient pu se produire : soit enrichir, soit apporter des filtres supplémentaires, notamment la construction de l'image de son propre corps, mais aussi la distanciation que l'on peut prendre quand de sujet d'étude vous devenez objet d'observation. Cette façon d'observer provoque un certain nombre de phénomènes et, par ailleurs, la façon d'analyser les choses va vous donner des pistes et notamment toutes les pistes qui sont dépendantes de l'observation des attitudes corporelles.

14 Je ne sais pas si se faire filmer et s'analyser par la suite est une pratique souvent utilisée par les anthropologues. Nous pouvons reprendre l'exemple célèbre de Margaret Mead et de Gregory Bateson à Bali où on ne voit jamais cette complexité. Mais c'était une époque où on considérait que la caméra appréhendait le réel sans être là. J'aurais du mal à trouver un exemple où l'anthropologue prend appui sur la caméra parce qu'il sait qu'elle est là et participe malgré tout pleinement à l'interaction sans en faire une sorte d'illustration ou de jeu. Je me dis que quand on se retrouve face à tant d'étrangeté (face à soi-même, notamment sous un angle de vue où l'on ne peut pas se voir soi-même, par exemple quand vous êtes pris de l'arrière), survient le problème de l'unité d'analyse : où 
doit-on découper temporellement et corporellement les éléments de base pour reconstruire les éléments de bases de l'interaction et de la négociation? Peut-être que, même sans aller dans ces problèmes techniques, le fait de se voir à la caméra permettrait une relance de l'écriture et du débat.

Alex Mucchielli : Il est vrai que si Yves Winkin avait été filmé par sa femme, il aurait eu un nouveau rôle parmi les quatre qu'il nous a déjà cité et il y aurait eu fixation d'un contexte prépondérant. Yves Winkin a très bien montré comment il revêtait quatre rôles différents au cours de quatre scènes différentes, je souhaiterais qu'il articule cette mise en scène avec le sens final que l'on pressent autour du thème de l'identité adhérée. Au cours de son expérience, Yves Winkin participe à plusieurs contextes: au contexte touristique occidental banal, dans la culture marocaine il tient un rôle ambivalent de riche et de pigeon, au contexte anthropologique, quand il s'observe en train de participer et donc est dans la culture anthropologique, enfin, il a sa propre identité à l'intérieur de laquelle se trouve le fameux affect, la honte, qui participe à d'autres contextes.

il nous montre que ces contextes agissent en même temps et finalement, il ne sait plus très bien où se situer, il se promène entre ces contextes; en définitive, son identité est altérée et il se demande quel rôle il a joué dans cette expérience. N’y a-t-il pas une articulation entre ces différentes couches de contextes? ]e plaide toujours pour la prépondérance du sens dans les phénomènes d'induction dans la mesure où cette intervention par les scènes ne ferait que renvoyer au sens final.

17 Je rajouterais un cinquième contexte temporalisé : renonciation. Ces différentes interventions s'étalent dans le temps. Chaque fois que je raconte cette histoire, je constate qu'elle a bougé, qu'il n'y a de sens final qu'au moment où je l'énonce. Vous parliez d'affect, c'est à ce niveau que je me sens en connivence avec Jeanne Favret-Saada. Cette histoire a dû sûrement m'affecter car j'y puise une énergie pour l'intellectualiser. J'ai besoin d'y revenir, d'où accumulation des sens en strates.

18 François Pichault : La première question à trait à la métaphore finale, celle du souffleur, qui me met un peu mal-à-l'aise. Pour moi, le souffleur est le gardien du texte. Or, je pense que l'exposé ne plaide pas pour cette forme de posture. J'ai tendance à raisonner sur l'expérience participante à partir de ma propre expérience dans le contexte organisationnel, contexte dans lequel nous avons le choix entre deux façons de mener l'expérience participante: une façon ouverte, déclarée comme telle, et une façon masquée, avec des écrits célèbres en sociologie de l'organisation. Si je suis dans la situation ouverte, déclarée comme telle, il n'y a pas de problème, je suis dans la salle. En revanche, si je suis dans la situation de l'observation masquée, se rajoute le problème de la duplicité qui s'introduit dans la relation avec ceux que je suis en train d'observer. La métaphore du souffleur ne me semble pas rendre compte de cette situation-là, situation délicate sur le plan déontologique, notamment dans le contexte de l'organisation.

La deuxième question porte sur la mémoire flottante: est-elle une rencontre entre la tentation typologique qui vient d'une démarche déductive, et un relativisme intégral auquel nous condamnerait une démarche strictement inductive?

La métaphore du souffleur est une façon de dire que l'observation va avoir un impact sur le texte énoncé, et dès le moment où il y a observation participante, on influe sur le jeu des acteurs de la scène. Mais la métaphore est trop lourde parce que ce n'est pas nous qui soufflons le texte aux acteurs, on dirait même que le souffleur a juste le droit de se taire. 
21 Il est vrai que dans le cas d'une relation masquée, la duplicité est un problème embarrassant. La seule façon dont la réparation peut se faire est au travers d'un autre débat qui donnera lieu à une relation écrite, à une publication et que le masque tombe. A mon avis, le chercheur doit, d'une manière ou d'une autre, trouver le moyen de réapparaître pour expliciter ce qu'il a tenté de faire. Il doit exister un moyen d'éviter les problèmes que certains chercheurs ont connus en travaillant totalement masqués, par exemple en explicitant au moins à une catégorie du personnel, ce que l'on va faire. Si quelques personnes le savent, c'est peut-être la meilleure façon pour le chercheur de s'en tirer ultérieurement.

En typologisant la démarche hypothético-déductive et le relativisme de l'induction complète, cette idée de maîtrise flottante est là pour dire que l'on est dans un cadrage du regard. Il n'y a pas de regard pur et innocent. Il faut qu'il soit orienté et fondé sur une théorie. Mais il ne faut pas non plus que ce cadrage soit à ce point dirigiste qu'il ne permette de voir que ce que l'on veut bien confirmer ultérieurement. Ici, la notion de la maîtrise flottante peut être utile.

23 Hassan Esmili : Je voudrais juste comprendre ce que vous entendez par «participer à la culture marocaine »?

Dans la mesure où les touristes, au Maroc, font partie des rôles, des acteurs, des personnages qui circulent, qui vont et viennent, et tout en étant à côté de la culture, font partie de la ville, j'étais dans un rôle reçu, connu, prévisible par les Marocains qui m'accueillaient. Ce n'est pas spécifique à la culture marocaine ; je n'occupais pas un point de vue extérieur, j'étais dedans, je participais à la culture, qui s'avérait cette fois-ci être la culture marocaine. Mais il n'y a pas de spécificité de la culture marocaine quant à la gestion du rapport au tourisme. Je crois que ce que j'ai vécu, j'aurais pu le vivre dans X cultures. 